\title{
Implementing Imaging Facilities and Multimedia in Teaching Veterinary Anatomy
}

\author{
A. S. Saber \\ Head of the Department of Anatomy \& Embryology, \\ Sadat City, Menoufiya University, EGYPT \\ E-mail : saberashraf 2@yahoo.com
}

\begin{abstract}
The fast waves of inventions and modifications of the high tech equipments in the last century converted the plane images into 2-dimensions and three-dimension ones. The appearances of the different multimedia facilities, as well as the prevalence of new pet animal species in the markets such as ostriches, Guinea pigs, hamsters, turtles, ..etc necessitate another and new conception in the morphology and anatomy teaching fields.

To reach the gross anatomy tutorial of the international level we have to follow the same steps and use the same tools and means used all over the world.
\end{abstract}

This proposal in the field of Veterinary Anatomy and Embryology may be a step on the way of developing the other fields of specialization in the Veterinary Medicine Profession. The developing of veterinary tutorial also matches the new global demand of accreditation and quality control programs adopted now in all our Universities and in other countries all over the world.

Imaging Facilities \& multimedia includes :

1) X-ray (1D image)

2) Digital radiography

3) Ultra-sonography

4) Computed Tomography (CT)

5) Magnetic Resonance Imaging (MRI)

6) Scintigraphy

7) Endoscopy

8) X-ray Stereo Fluoroscopy

9) Thermography

10) Other Techniques.

a- Plastination

b- Animation

c- Skeletons display.

\section{1) X-ray}

\section{What is X-ray?}

$X$-ray is the most commonly performed diagnostic method of examination. Approximately half of all $x$-rays obtained in medical institutions are chest $x$ rays. An x-ray is usually done for the evaluation of lungs, heart and chest wall. Pneumonia, heart failure, emphysema, lung cancer and other medical conditions (fractures, lameness, swellings, swallowed foreign bodies ..etc) can be diagnosed or suspected on an $\mathrm{x}$-ray image.

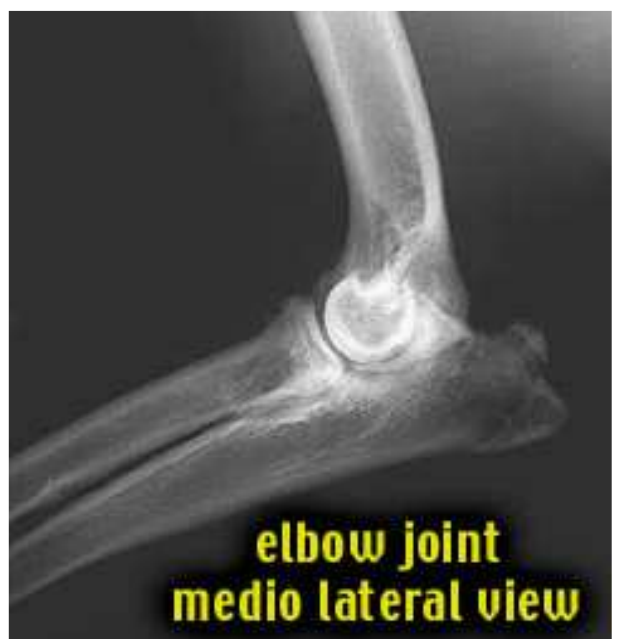

$X$-ray images for the elbow joint of dog 


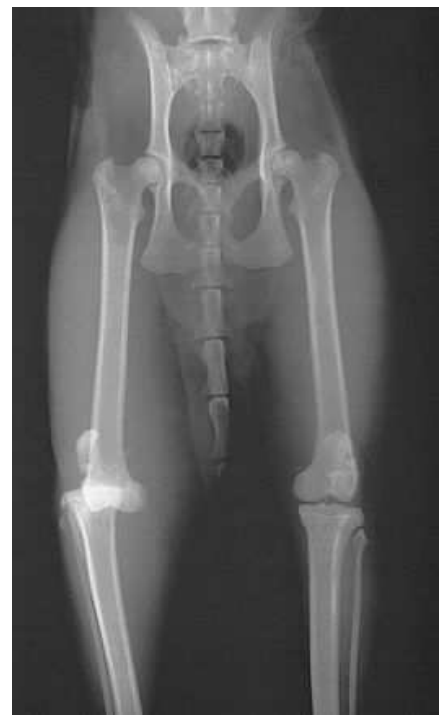

$X$-ray image of the pelvic limbs of a dog

\section{Micro-X-Ray Fluorescence (MXRF).}

When a surface is exposed to a thin beam of $x-$ rays, the MXRF instrument detects elements such as sodium, potassium and chlorine, which are present as salts in human sweat. Because the salts are deposited along the ridges present in a fingerprint, the fluorescence can be used to assemble a digital image of a print.

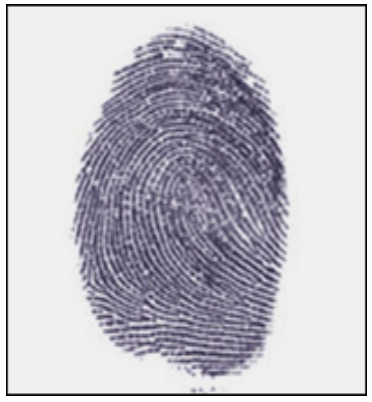

The use $X$-Rays to 'See' Fingerprints (a recent method invented in April, 2005 to be used in forensic medicine)

\section{(2) Digital Radiography}

\section{Computed Radiography Systems :}

With this equipment, digital medical images of sick or injured animals can be captured in seconds and transmitted to radiologists, veterinary specialists and other professionals for immediate diagnosis and consultation.

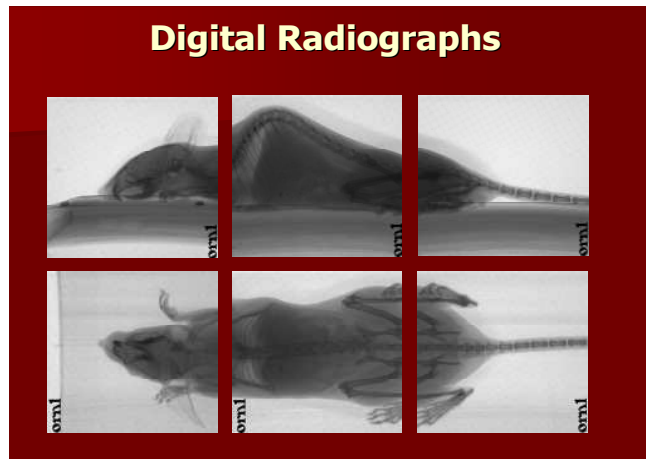

\section{(3) Ultra Sonography}

\section{What is an Ultrasound?}

Ultrasound is the use of high frequency sound waves to create images from within the body. By using these sound waves, images can be obtained of a variety of internal organs and structures.

Ultrasound is utilized in studies of the major organs, the heart, blood vessels, and of course, in obstetrics where the unborn fetus can be observed.

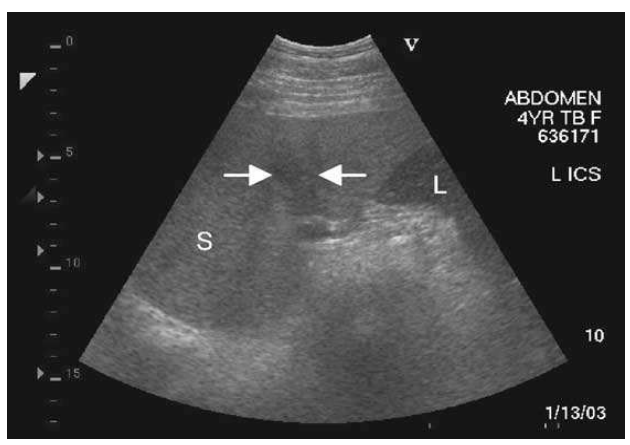

Abnormal spleen. An irregularly shaped hypoechoic area (arrows) is seen within the splenic parenchyma in a 4-yr-old horse

\section{(4) Computer Tomography (CT)}

What is Computed Tomography (CT Scan)? 
Computed tomography (CT), and sometimes called a CAT scan, uses special x-ray equipment to obtain images from different angles around the body. Those images are then run through a computer to produce a cross-section of body tissue and organs.

\section{How does CT work?}

CT units produce a very thin fan of $x$-rays which are directed through the patient and strike a row of radiation detectors. The amount of radiation going through a specific part of the patient, and therefore reaching the detector is related to the density of the body part. A CT scanner takes numerous views of each part, and reconstructs an image based on the density of different areas.

\section{What are the indications for CT?}

CT is particularly useful for looking at complex bony structures such as the skull, spine or joints. It is also useful for detecting and characterizing lung diseases. Both CT and MRI are excellent for assessing blood flow to an organ or region.
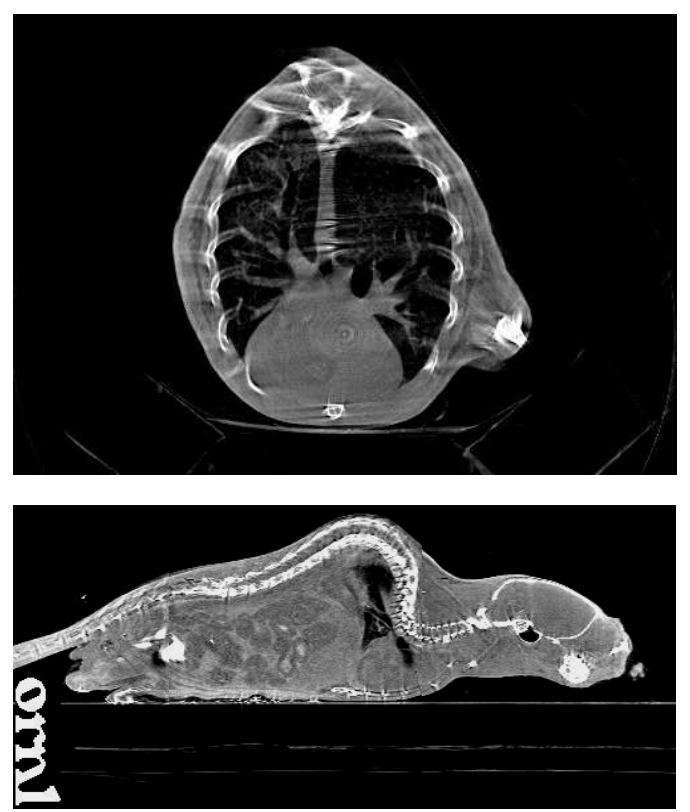

View : Transverse image of a mouse thorax(upper) Notes: 3 week old male white mouse with IP injected iodine contrast agent.

View : Sagittal (lower)

Notes: 3 week old male white mouse with IP injected iodine contrast agent.

J. vet. anat.

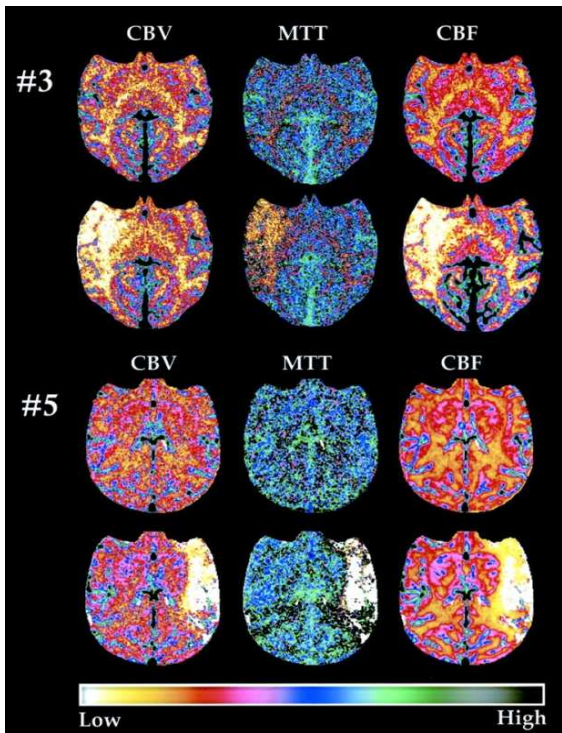

Functional CT Perfusion Imaging

\section{(5) Magnetic Resonance Imaging (MRI)}

What is Magnetic Resonance Imaging (MRI)? Magnetic resonance imaging (MRI) uses radiofrequency waves and a strong magnetic field rather than x-rays to provide remarkably clear and detailed pictures of internal organs and tissues.

\section{How does MRI work?}

When a patient is put in a strong magnet, some of the atoms become aligned with the magnetic field. If a carefully tuned radio-frequency (RF) pulse is sent into the patient, those atoms can be tipped over. As the atoms realign themselves with the magnetic field, they give off an RF pulse that can be detected by the MRI scanner. The amount of RF signal given off, and the time at which it is released are characteristic for certain tissues. RF signal changes can differentiate normal from abnormal tissues such as those affected by cancer, infection or trauma.

\section{What are the indications for MRI ?}

MRI is useful for looking at soft tissue structures which have low contrast on conventional radiographs (x-rays) and complex soft tissue structures. The largest indications are in imaging the brain, spinal cord, and soft tissues of the musculoskeletal system. 


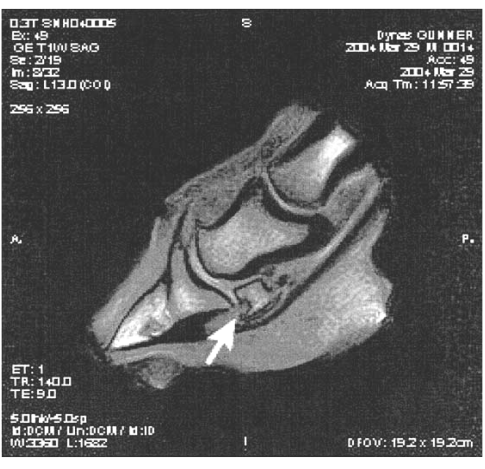

Standing MRI of the equine foot. Sagittal view,

\section{(6) Scintigraphy}

- Scintigraphy: Is a diagnostic test in which a two-dimensional picture of a body radiation source is obtained through the use of radioisotopes.

- Nuclear scintigraphy is classified as a metabolic imaging modality, because it relies on the inherent living properties of the tissue to produce an image .

- A bone seeking agent, usually methylene diphosphonate (MDP), is labeled with a radioactive compound (technetium $99 \mathrm{~m}$ ) to produce a radiopharmaceutical .

- This is injected intravenously; then, imaging commences
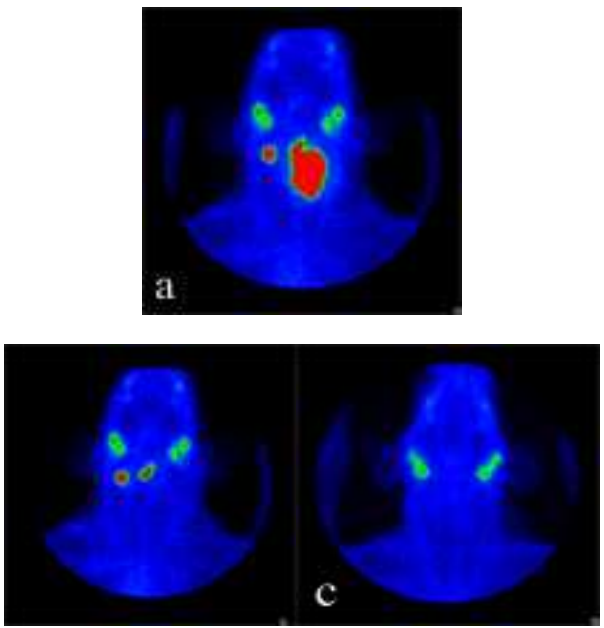

Planar thyroid scintigraphy of a hyperthyroid dog with a hyperfunctional thyroid carcinoma at presentation (a), post operatively (b) and after ablative radioiodine therapy $(c)$.

J. vet. anat.

\section{(7) Endoscopy}

Endoscopy is a technique that allows examination of an area of the body by means of an endoscope, a tube-like instrument with lenses and a light source attached. The endoscope provides visual examination of the interior of the body through a natural body opening such as the throat or through a small incision into the body.
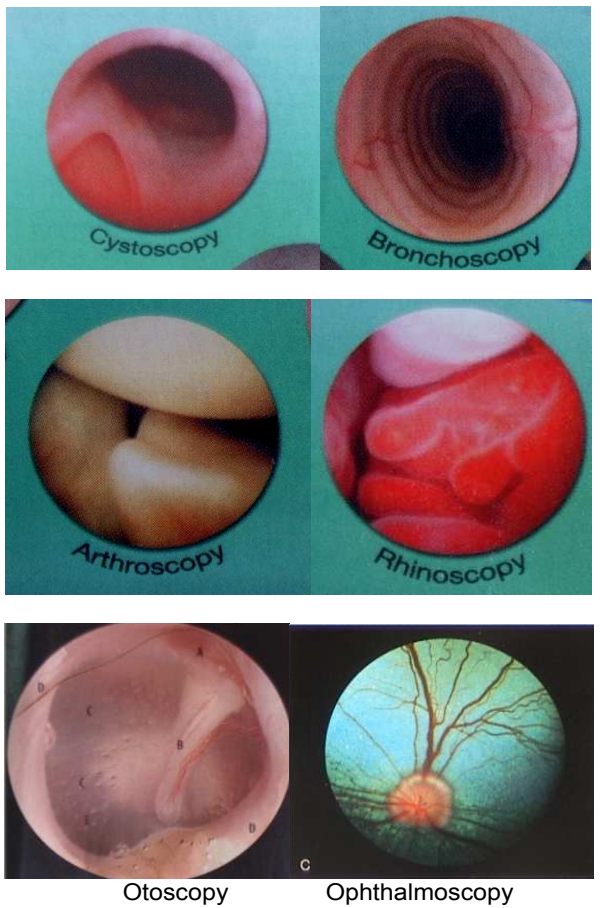

\section{(8) X-ray Stereo Fluoroscopy}

Fluoroscopy is an imaging technique that uses $\mathrm{X}$ ray to produce real-time or "live" images of organs and anatomical structure motion.

Fluoroscopy is primarily used in barium studies of the gastrointestinal tract and in IV contrast studies of the urinary tract and the arterial system. 


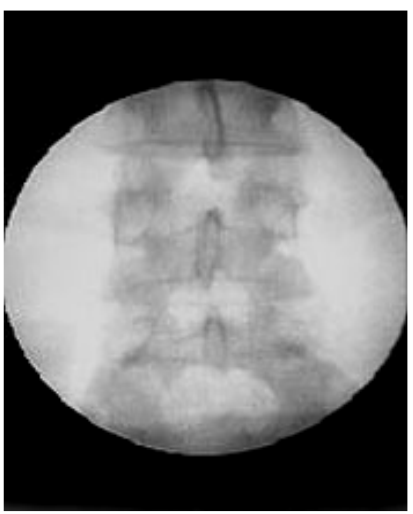

Image of what a fluoroscope sees

\section{(9) Thermography (Physiologic Image)}

* Recent advances in infrared imaging allow us to use this technology to assist in lameness cases.

* The equipment ( infrared detector camera) measures minute changes in temperature at the skin surface and reports this information as a color-encoded photograph.

* Recent advances in infrared imaging allow us to use this technology to assist in lameness cases.

* The equipment ( infrared detector camera) measures minute changes in temperature at the skin surface and reports this information as a color-encoded photograph.

* The camera is, in fact, an infrared detector. All living creatures give off infrared heat, and inflammation means greater heat than normal over injured areas. Decreased heat can mean injury, too.

* The white image represents the hottest temperature, the blue the coolest. As it goes to shades of green to yellow to red to white, heat increases.

* Thermography can detect temperature differences of less than one-half of a degree.

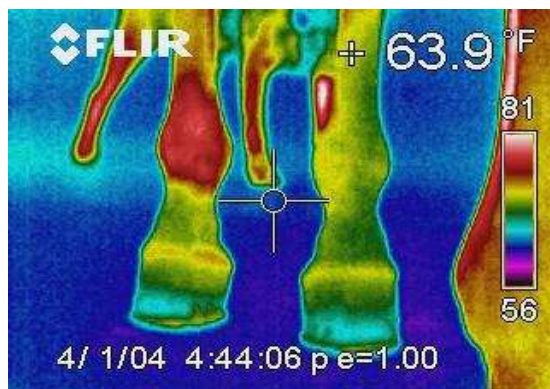

Thermography of horse legs

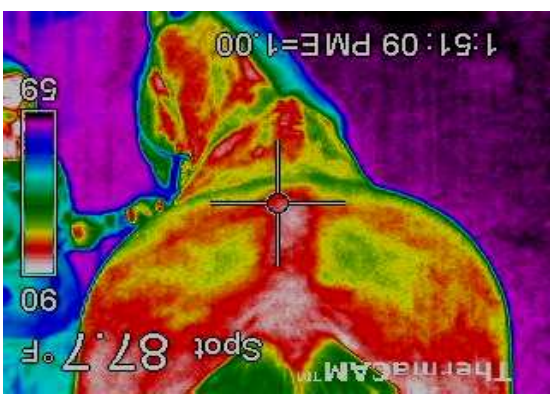

Thermography of the back of a horse

\section{(10) Other techniques}

\section{A) Plastination}

Is a relatively new method of preserving and producing anatomical specimens free from smell (formalin), easy to be handled and to be used for a long time.

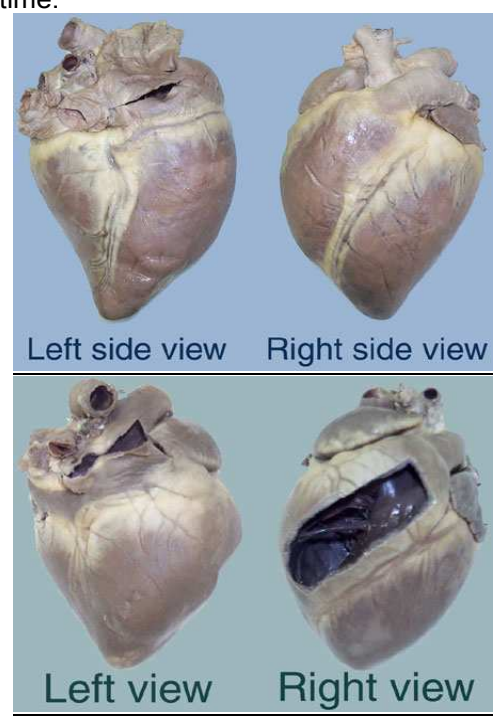

\section{B)Animation}

The development of computer programs and the skills of the programmers lead to production of programs for animation describing and clearing many facts in the animal bodies which made them easy to imagine and understand. 

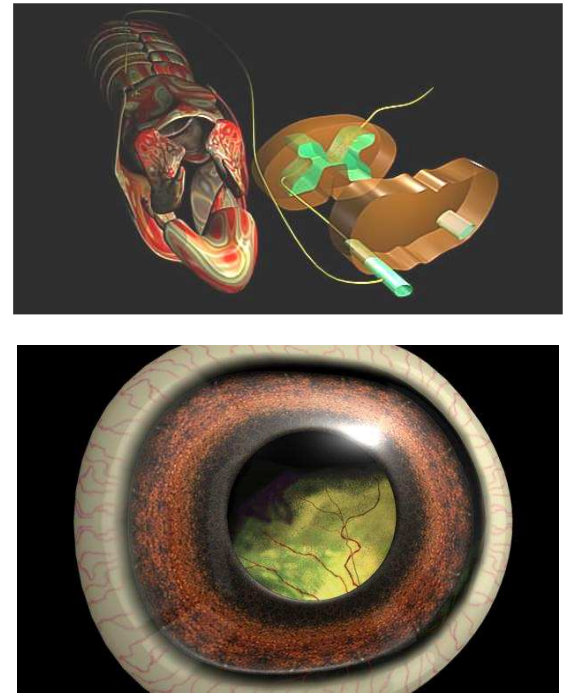

horse larynx, from an animation canine visual lesions

\section{C)Skeletons Display}

Although a very old method for studying anatomy, animals models and skeleton is still used and is an indispensable method of learning anatomy.

\section{Conclusion}

- The involvement of the new techniques ( Digital X-ray images, Computed Tomography (CT) Magnetic Resonance Images (MIR), Ultrasonography, Endoscopy, Fluoroscopy, 3D Image, animation, CDs...etc ) in the anatomy tutorial in Egypt, as well as in the other Arabic countries becomes essential to follow the international steps and to prepare the students to the clinical stage of veterinary study.

- The need to include the applied anatomy either in the anatomy tutorial or as a separate course for the clinical phase students becomes necessary .

- Some wild animal species( amphibian, birds and mammal ) needs to be studied by the postgraduates.

\section{References}

www.flirthermography.com

www.infraredtraining.com

http://www.medicinenet.com

http://en.wikipedia.org/wiki/Scintigraphy

Medical Imaging : Techniques Reflection \&

Evaluation

by Elizabeth Carver (Author), Barry Carver (Editor) 2006.

Pattern Recognition for Medical Imaging

by Anke Meyer-Base

ISBN: 9780124932906

Modalities

by Cornelius T Leondes (Editor)

ISBN: 978981256992 